\section{POS0543 MORNING STIFFNESS IN CLINICALLY SUSPECT ARTHRALGIA IS EXPLAINED BY LOCAL SUBCLINICAL SYNOVITIS AND SYSTEMIC INFLAMMATION}

D. Krijbolder ${ }^{1}$, F. Wouters ${ }^{1}$, E. van Mulligen ${ }^{2}$, A. van der Helm - van Mil ${ }^{1}$.2. ${ }^{1}$ Leiden University Medical Centre, Department of Rheumatology, Leiden, Netherlands; ${ }^{2}$ Erasmus Medical Center, Department of Rheumatology, Rotterdam, Netherlands

Background: Morning stiffness (MS) is considered a cardinal symptom in the clinical appraisal of arthralgia patients, suggesting presence of subclinical inflammation, which could indicate an increased chance of progression to rheumatoid arthritis (RA). However, the pathophysiology behind MS in arthralgia patients that is clinically suspect for progressing to RA (clinically suspect arthralgia; CSA) has never been studied. In RA, it is presumed that both and local- and systemic inflammation underlie MS. We therefore hypothesize that, in patients with CSA, MS can also be explained by local- and systemic inflammation.

Objectives: To determine if MS can be explained by MRI-detected local inflammation (subclinical synovitis and tenosynovitis) and systemic inflammation (C-reactive protein(CRP)).

Methods: 514 CSA patients underwent a contrast-enhanced 1.5T MRI of metacarpophalangeal (MCP) 2-5, wrist and metatarsophalangeal (MTP) 1-5 joints, next to clinical assessment and laboratory investigations. MRIs were scored for synovitis and tenosynovitis in line with the RAMRIS-method. MS was dichotomized as present (duration $\geq 60$ minutes) or absent (duration $<60$ minutes). Associations of MRI-detected synovitis, tenosynovitis and increased CRP with MS were tested with univariable and multivariable logistic regression. Since earlier research in arthritis patients showed that the effect of combined presence of MRI-detected synovitis and tenosynovitis was increased, compared to the effect of these features separately, interaction between MRI-detected synovitis and tenosynovitis, and between synovitis and increased CRP, was assessed.

Results: In the studied CSA-patients, mean age was 44 years (sd 13), 397 patients $(77 \%)$ were female, median tender joint count (TJC-70) was 5 (interquartile range 2-10), and 67 (13\%) patients were ACPA-positive. MS was present in 191 (37\%) CSA-patients. Baseline characteristics among patients with and without MS were similar. MRI-detected synovitis was more often present in patients with MS compared to patients without MS (34\% versus 19\%), OR 2.12 (95\% Cl 1.41-3.19). Also, MRI-detected tenosynovitis was more frequently present in patients with MS $(36 \%$ versus $24 \%)$, OR 1.74 (1.18-2.57). Likewise, increased CRP levels ( $\geq 5 \mathrm{mg} / \mathrm{L})$ were more often found in patients with MS (31\% versus 18\%), OR 2.00 (1.32-3.04). In multivariable analyses, ORs were 1.90 (1.22-2.96) for MRI-detected synovitis and 1.82 (1.18-2.82) for increased CRP. With an OR of 1.20 (0.77-1.87) MRI-detected tenosynovitis was not significantly associated with MS in a multivariable analysis. Interaction between synovitis and tenosynovitis, and between synovitis and CRP was not significant ( $p$-value of 0.13 and 0.15 , respectively).

Conclusion: Presence of MRI-detected synovitis and increased CRP levels are associated with presence of MS in patients with CSA. This indicates that MS in CSA patients could indeed be induced by both local- and systemic inflammation. Disclosure of Interests: None declared

DOI: 10.1136/annrheumdis-2021-eular.2474

\section{POS0544 INFLUENCE OF EATING HABITS ON FRAILTY AMONG PATIENTS WITH RHEUMATOID ARTHRITIS: KURAMA COHORT}

M. Katsushima ${ }^{1}$, H. Minamino ${ }^{2}$, M. Torii ${ }^{3}$, M. Hashimoto ${ }^{4}$, W. Yamamoto ${ }^{5}$, R. Watanabe ${ }^{4}$, K. Murakami ${ }^{1}$, K. Murata ${ }^{4}$, M. Tanaka ${ }^{4}$, H. Ito ${ }^{4}$, A. Morinobu ${ }^{1} .{ }^{1}$ Kyoto University Graduate School of Medicine, Department of Rheumatology and Clinical Immunology, Kyoto, Japan; ${ }^{2}$ Kyoto University Graduate School of Medicine, Department of Diabetes, Endocrinology and Nutrition, Kyoto, Japan; ${ }^{3}$ Kyoto University Graduate School of Medicine, Department of Human Health Sciences, Kyoto, Japan; ${ }^{4}$ Kyoto University Graduate School of Medicine, Department of Advanced Medicine for Rheumatic Diseases, Kyoto, Japan; ${ }^{5}$ Kurashiki Sweet Hospital, Department of Health Information Management, Kurashiki, Japan

Background: Rheumatoid arthritis (RA) is a chronic inflammatory disorder that contributes to accelerating frailty, a clinical state of increased vulnerability due to declined physiological function. Although accumulating evidence suggests the importance of nutritional therapy for frailty in the general population, there is little evidence on dietary recommendations for preventing frailty in patients with RA. Objectives: The present study aimed to reveal clinical associations between frailty status, eating habits and RA disease activity.

Methods: We conducted a cross-sectional study of 306 female outpatients enrolled from the KURAMA (Kyoto University Rheumatoid Arthritis Management Alliance) cohort database. The participants were classified into three groups (robust, prefrail and frail) according to simplified frailty scale (SOF index), and dietary data were collected using a self-reported food frequency questionnaire as previously reported. We performed multivariate logistic analyses for the presence of frailty/prefrailty with or without eating habits.
Results: Frail group showed physical decline such as decreased skeletal muscle index, hand grip strength and walking speed, and DAS28-ESR in the frail group was significantly higher compared to that in the others. In multivariate logistic analysis, the presence of frailty/prefrailty was correlated with DAS28-ESR (OR 1.71 $p=0.00004$ ) and methotrexate use (OR $0.47, p=0.0097$ ). Cochran-Armitage trend test also showed that the intake frequency of five ingredients (meat, fish, milk, fruits and vegetables) was inversely associated with the prevalence of frailty/prefrailty. In additional multivariate logistic analyses with dietary habits, habitual intake of fish (at least three times per week), rather than meat or other foods, was independently correlated with the presence of frailty/prefrailty (OR $0.33, p=0.00027$ ).

Conclusion: Our results suggest that habitual intake of fish, rather than meat or other foods, may be beneficial in preventing frailty among RA patients.

REFERENCES:

[1] Ferrucci, L. \& Fabbri, E. Inflammageing: chronic inflammation in ageing, cardiovascular disease, and frailty. Nat Rev Cardiol 15, 505-522, doi:10.1038/ s41569-018-0064-2 (2018).

[2] Hernandez Morante, J. J., Gomez Martinez, C. \& Morillas-Ruiz, J. M. Dietary factors associated with frailty in old adults: a review of nutritional interventions to prevent frailty development. Nutrients 11, doi:10.3390/nu11010102 (2019).

Table 1. Multivariate logistic analysis for RA patients with prefrailty or frailty

\begin{tabular}{|c|c|c|c|c|}
\hline \multirow{2}{*}{$\begin{array}{l}\text { variables including } \\
\text { eating habits }\end{array}$} & \multicolumn{2}{|c|}{ Fish + Meat } & \multicolumn{2}{|l|}{ All } \\
\hline & OR $(95 \%$ Cl) & $P$ value & OR $(95 \% \mathrm{Cl})$ & $P$ value \\
\hline DAS28-ESR & $1.78(1.34-2.37)$ & 0.00003 & $1.73(1.30-2.30)$ & 0.00009 \\
\hline MTX use & $0.43(0.23-0.79)$ & 0.0055 & $0.42(0.23-0.78)$ & 0.0050 \\
\hline Age (1 year) & $1.02(1.00-1.05)$ & 0.037 & $1.03(1.01-1.06)$ & 0.0015 \\
\hline PSL use & $1.23(0.69-2.21)$ & 0.49 & $1.22(0.67-2.20)$ & 0.51 \\
\hline Duration of RA (1 year) & $1.00(0.98-1.02)$ & 0.72 & $1.00(0.98-1.02)$ & 0.84 \\
\hline Body mass index & $1.00(0.93-1.07)$ & 0.98 & $0.99(0.92-1.07)$ & 0.85 \\
\hline Biological agents use & $1.02(0.60-1.72)$ & 0.94 & $1.04(0.62-1.77)$ & 0.87 \\
\hline Fish dish & $0.31(0.17-0.55)$ & 0.00004 & $0.33(0.18-0.61)$ & 0.00027 \\
\hline Meat dish & $0.86(0.49-1.50)$ & 0.60 & $0.89(0.51-1.57)$ & 0.69 \\
\hline Milk & & & $0.71(0.41-1.24)$ & 0.23 \\
\hline Vegetable & & & $0.95(0.47-1.93)$ & 0.89 \\
\hline Fruits & & & $0.77(0.41-1.42)$ & 0.40 \\
\hline
\end{tabular}

A$$
\text { व }
$$

递

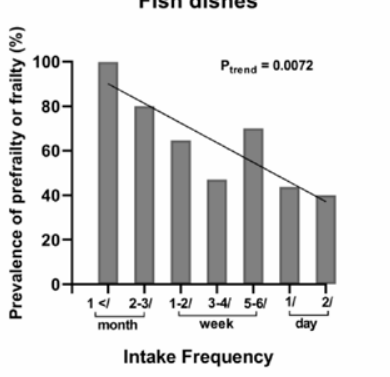

C

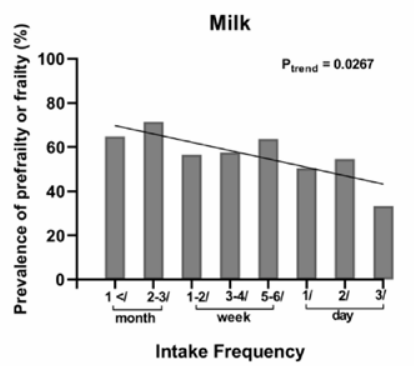

E

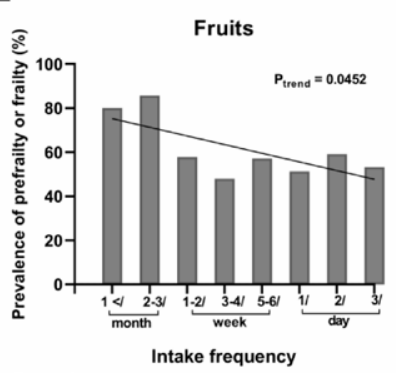

B

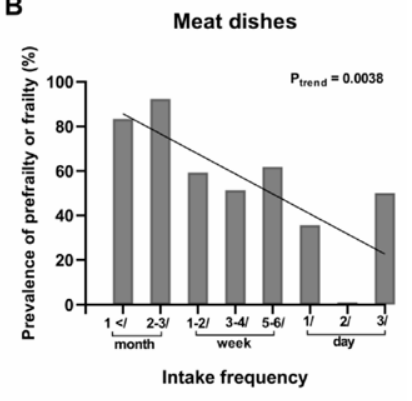

D

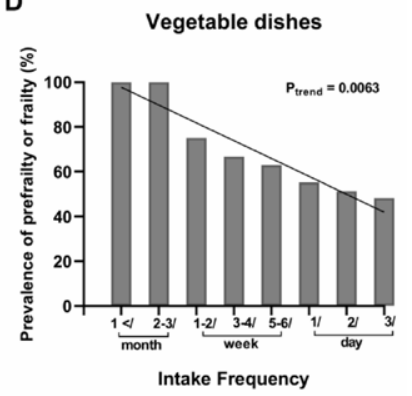

Figure 1. The prevalence of prefrailty or frailty for subjects by intake frequency 